\title{
High-fat feeding stimulates endocrine, glucose-dependent insulinotropic polypeptide (GIP)-expressing cell hyperplasia in the duodenum of Wistar rats
}

\author{
D. Gniuli $\cdot$ A. Calcagno $\cdot$ L. Dalla Libera $\cdot$ R. Calvani $\cdot$ \\ L. Leccesi • M. E. Caristo $\cdot$ R. Vettor $\cdot$ M. Castagneto • \\ G. Ghirlanda • G. Mingrone
}

Received: 23 April 2010 /Accepted: 24 May 2010 / Published online: 30 June 2010

(C) Springer-Verlag 2010

\begin{abstract}
Aims/hypothesis Incretins are hormones released by enteroendocrine cells in response to meals, depending upon absorption of nutrients. The present study aimed to elucidate the mechanisms through which a high-fat diet (HFD) induces insulin resistance and insulin hypersecretion by focusing on the effects on enteroendocrine cells, especially those secreting glucose-dependent insulinotropic polypeptide (GIP).

Methods Forty male Wistar rats, 4 months old, were randomised into two groups; one group received a chow
\end{abstract}

D. Gniuli $\cdot$ R. Calvani $\cdot$ L. Leccesi $\cdot$ G. Ghirlanda •

G. Mingrone $(\triangle)$

Department of Internal Medicine and Diabetes Unit,

Università Cattolica del Sacro Cuore,

L.go Gemelli 8,

00168 Roma, Italy

e-mail: gmingrone@rm.unicatt.it

A. Calcagno $\cdot$ R. Vettor

Department of Medical and Surgical Sciences, Internal Medicine,

University of Padua,

Padua, Italy

M. Castagneto

Department of Surgery, Università Cattolica del Sacro Cuore,

Roma, Italy

L. Dalla Libera

Unit of Neuromuscular Biology and Pathophysiology,

Department of Biomedical Sciences,

CNR Institute of Neurosciences, University of Padua,

Padua, Italy

M. E. Caristo

Department of Animal House, Università Cattolica S. Cuore,

Roma, Italy diet and the other one received a purified tripalmitin-based HFD ad libitum. An OGTT was performed every 10 days and histological and immunofluorescence evaluations of the duodenum were obtained at 60 days from the beginning of the diets. Plasma glucose, insulin, GIP and glucagon-like peptide-1 (GLP-1) levels were measured. Immunofluorescence analysis of duodenal sections for pancreatic duodenal homeobox-1 (PDX-1), KI67, GLP-1, GIP and insulin were performed.

Results Compared with chow diet, HFD induced a progressive significant increase of the glucose, insulin and GIP responses to OGTT, whereas GLP-1 circulating levels were reduced over time. After 60 days of HFD, cellular agglomerates of KI67 and PDX-1 positive cells, negative for insulin and GLP-1 but positive for GIP staining, were found inside the duodenal mucosa, and apoptosis was significantly increased.

Conclusions/interpretation With the limitation that we could not establish a causal relationship between events, our study shows that HFD stimulates duodenal proliferation of endocrine cells differentiating towards $\mathrm{K}$ cells and oversecreting GIP. The progressive increment of GIP levels might represent the stimulus for insulin hypersecretion and insulin resistance.

Keywords Duodenum · Glucose-dependent insulinotropic polypeptide - Glucagon-like peptide-1 - Endocrine cell hyperplasia $\cdot$ High-fat diet . Insulin

$\begin{array}{ll}\text { Abbreviations } \\ \text { GIP } & \text { Glucose-dependent insulinotropic polypeptide } \\ \text { GLP-1 } & \text { Glucagon-like peptide-1 } \\ \text { HFD } & \text { High-fat diet } \\ \text { PDX-1 } & \text { Pancreatic duodenal homeobox-1 }\end{array}$




\section{Introduction}

The deranging effects of a high-fat diet (HFD) on beta cells, insulin secretion and insulin resistance have been extensively evaluated and documented by several research groups, including ours [1-3]. However, how HFD induces insulin resistance and diabetes is still unclear, and the major hypothesis that damage to mitochondria by fatty acids can be the primum movens in inducing glucose metabolism alteration has been disproved [4]. In fact, although 1 month of HFD was proved to be sufficient to induce glucose intolerance in mice, no evidence of mitochondrial dysfunction was found and an altered mitochondrial biogenesis, structure, and function in muscle tissue was observed only after the manifestation of frank diabetes [5].

High-saturated fat feeding is a nongenetic factor affecting insulin sensitivity, but the factors that determine the ability of beta cells to compensate for insulin resistance, and the mechanisms involved, remain largely unknown.

High-fat feeding induces $\mathrm{K}$ cell hyperplasia and glucosedependent insulinotropic polypeptide (GIP) secretion [6-9] with insulin secretion potentiating effects [10]. Blockade of GIP action by administration of a specific GIP receptor antagonist, $\left(\right.$ Pro $\left.^{3}\right)$ GIP, reverses the glucose intolerance induced by HFD [11] without affecting circulating insulin concentration.

Glucagon-like peptide-1 7-36 (active form; GLP-1 ${ }_{7-36}$ ) acts to potentiate glucose-induced insulin secretion, inhibit glucagon secretion, decrease food intake, improve insulin sensitivity and may also promote beta cell proliferation [12]. Simarly to GIP receptor antagonist, the GLP-1 receptor agonist (D-Ala(8))GLP-1 is effective in the treatment of glucose intolerance and insulin resistance caused by high-fat feeding [13].

However, the mechanisms through which HFD stimulates GIP secretion and induces insulin resistance and insulin hypersecretion remain to be clarified.

In the present study we investigated the effects of a subchronic exposure to HFD in Wistar rats on enteroendocrine cells, especially GIP-secreting cells, and the relationship with insulin resistance and insulin secretion.

\section{Methods}

Experimental animals Forty male Wistar rats obtained from an in-house breeding colony were randomised at about 4 months of age into two groups: one group received a control $\operatorname{diet}(3.2 \%$ fat containing $1.3 \%$ tripalmitin) ad libitum and the other group received a purified tripalmitin-based HFD ad libitum. Both diets (Rieper AG SpA, Bolzano, Italy) contained corn oil $(1.9 / 100 \mathrm{~g}$ diet $)$ to prevent essential fatty acid deficiency. The HFD supplied $59 \%$ of energy as fat and $20 \%$ as carbohydrate comprised of cornstarch and sucrose $(2: 1 \mathrm{wt} / \mathrm{wt})$. The control diet provided $10 \%$ of energy as fat and $65 \%$ as carbohydrates.

The energy density of the diets was $13.81 \mathrm{~kJ} / \mathrm{g}$ for the chow diet and $21.10 \mathrm{~kJ} / \mathrm{g}$ for HFD.

The animals were killed with an overdose of pentobarbital $(200 \mathrm{mg} / \mathrm{kg}$ i.p.) after an overnight fast at 60 days after the beginning of either control diet or HFD.

The control diets or HFDs were continued for 60 days. All animals underwent an OGTT basally and every 10 days until 60 days after the beginning of the diet. All animals had free access to water and were subjected to controlled temperature $\left(22 \pm 1{ }^{\circ} \mathrm{C}\right)$ and lighting (lights on $06: 00$ to 18:00 hours). The experimental procedures performed were approved by the Catholic University Animal Experimentation Ethics Committee and were in accordance with European guidelines for the use of animals in research

OGTT Animals were fasted overnight and then received a $50 \%$ D-glucose solution ( $1 \mathrm{~g} / \mathrm{kg}$ body weight $)$ by oral gavage. Blood was collected from the tail for measurement of glucose and insulin concentrations at $0,5,15,30,45,60$, $90,120,150$ and $180 \mathrm{~min}$ before starting the diet and 60 days after the beginning of the diet. Every 10 days after the beginning of the diet, i.e. at 10, 20, 30, 40 and 50 days, an OGTT was perfomed and blood samples were collected at baseline and at 30 min to measure peak plasma glucose and insulin. A second aliquot of blood was placed in tubes containing EDTA, aprotinin and a dipeptidyl peptidase-IV inhibitor, and analysed for GLP-1 $1_{7-36}$ and total GIP.

After centrifugation, plasma was divided into appropriate subsamples and stored at $-20^{\circ} \mathrm{C}$ until analysis.

Analytical methods Plasma glucose levels were analysed by the glucose-oxidase method (Glucose Analyzer II; Beckman, Fullerton, CA, USA). Serum insulin, plasma GLP-1 ${ }_{7-36}$ and total GIP were measured by rodent/rat-specific ELISAs (Millipore, St Charles, MO, USA): for GIP: sensitivity $8.2 \mathrm{pg} / \mathrm{ml}$, inter-assay $1.1-5.9 \%$, intra-assay $1.0-5.9 \%$, accuracy $104.2 \pm 4.8 \%$; and for GLP- $1_{7-36}$ : sensitivity $5.2 \mathrm{pg} / \mathrm{ml}$, intra-assay $<11 \%$, inter-assay $<19 \%$, accuracy $83 \%$.

Tissue fixation, histology and immunofluorescence analysis Duodenum was removed from all rats and weighed. Part was fixed overnight in $10 \%$ formalin in PBS and serial $5 \mu \mathrm{m}$ thick sections were cut on a cryostat and floated onto slides for immunofluorescence staining and TUNEL analysis.

Fixed tissues were then routinely processed for paraffin embedding and $4 \mu \mathrm{m}$ thick sections were cut for haema- 
toxylin and eosin staining to compare intestinal villi before and after HFD.

Cellular proliferation Cellular proliferation was evaluated using the cell cycle antigen KI67 (NCL-Ki67p; Novocastra, Newcastle upon Tyne, UK). Human KI67 nuclear antigen expresses on all proliferating cells during late G1, S, M and G2 phases of the cell cycle and crossreacts with rat and mouse KI67 nuclear antigen. After incubation, binding of primary antibody was detected with Alexa Fluor 594 goat anti-mouse secondary antibodies (Molecular Probes, Eugene, OR, USA; and Invitrogen, Carlsbad, CA, USA). Nuclei were counterstained with Hoechst 33258 (SigmaAldrich, St Louis, MO, USA) and mounted for examination with mounting media for fluorescence (Kirkegaard \& Perry Laboratories, Gaithersburg, MD, USA). For localisation of fluorescence, specimens were examined and photographed on a Diaphot microscope (Nikon, Brussels, Belgium) equipped with phase-contrast and epifluorescence optics $(\times 100)$ lens. Pictures were recorded on Kodak Gold Plus film (Eastman Kodak).

Control experiments were carried out to check the specificity of the antibody binding by omitting the primary antibody.

PDX-1-positive cells For detection of pancreatic duodenal homeobox-1 (PDX-1), tissues were prepared as before. An affinity-purified goat polyclonal antibody was used, raised against a peptide mapping within an internal region of PDX-1 of human origin (sc-14664 P; Santa Cruz Biotechnology, Santa Cruz, CA, USA). PDX-1 (A-17) antibody dilution was 1:500.

The secondary antibody was an Alexa Fluor 594 rabbit antigoat secondary antibody (Molecular Probes, Invitrogen). Control studies were performed using the above methods with deletion of individual primary antisera.

GIP-positive cells For GIP detection, sections were incubated overnight at $4^{\circ} \mathrm{C}$ with a primary antibody (GIP:sc23554; Santa Cruz Biotechnology, Inc.), washed, then incubated with secondary antibody (Alexa 488 donkey anti-goat antibody), incubated with TOPRO-3 (Molecular Probes) for nuclear staining, washed, mounted with fluorescence mounting medium (Vectashield; Vector Laboratories, Burlingame, CA, USA) and photographed as previously described.

GIP antibodies were used at a dilution of 1:500.

GLP-1-positive cells For GLP-1 detection, sections were incubated with a primary antibody (GLP-1:sc-7782; Santa Cruz Biotechnology); the GLP-1 (HYB 011-05) dilution was 1:200.
The sections were washed and incubated with secondary antibody (Alexa 488 donkey anti-goat antibody) and then we proceeded as reported above.

Evaluation of insulin-producing cells Slides were immunostained for insulin to exclude the possibility that PDX-1positive and KI67-positive cells could produce insulin. A mouse monoclonal anti-insulin antibody (NCL-INSULIN; Novocastra) was used at a dilution of 1:100 in PBS for double-staining with anti-KI67 and anti-PDX-1. Slides were then exposed to a secondary Alexa Fluor 488 goat anti-mouse secondary antibody (Molecular Probes, Invitrogen). Frames were captured and analysed as previously described.

TUNEL and nuclear condensation Tissue preparations were obtained as described earlier. Terminal deoxynucleotidyl transferase enzyme and dUTP conjugated to a fluorescein cocktail were added to the tissue sections according to the manufacturer's specifications (Boehringer Mannheim in situ death detection kit; Boehringer Mannheim, Monza, Italy). Nuclei were counterstained with Hoechst 33258 (Sigma-Aldrich). Speciments were mounted for examination with mounting media for fluorescence, examined and photographed as previously described for immunofluorescence evaluation.

The total number of positive nuclei was determined by counting (at a magnification $\times 250$ ) all of the labelled nuclei in the whole specimen. The number of positive nuclei $(n=5$ duodena per point) was then expressed as the number of TUNEL-positive nuclei per $\mathrm{mm}^{3}$.

The ELISA for the apoptosis DNA ladder assay was performed according to Yasuhara et al. [13] as previously described [14]. Cell death ELISA analysis was performed according to the manufacturer's instructions (Boehringer Mannheim). After incubation, the plates were analysed with a multiwell ELISA reader.

Statistical analysis Values were expressed as mean \pm SEM. The incremental AUC was computed using the trapezoidal rule. OGTT data were compared by two-factor (time $\times$ treatment) repeated measures ANOVA followed by post hoc analysis with Bonferroni's multiple comparison test. Other variables were analysed by Student's $t$ test. Differences were considered significant at $p<0.05$.

After preliminary Pearson correlation, a linear multiple regression analysis was performed to detect the best predictors of repeated plasma glucose concentrations at the peak after the OGTTs during the high-fat feeding period as well as partial and part correlations.

Cross-correlation analysis was performed to check for correlations between per cent increment of GIP and insulin 
peaks over the basal values after OGTT during the 60 day observation period.

\section{Results}

Body weight At the beginning of the study the average weight of control rats was $238.3 \pm 15.1 \mathrm{~g}$ and that of the HFD rats was $244.3 \pm 12.1 \mathrm{~g}(p=\mathrm{NS})$, increasing to $360.2 \pm 28.2$ and $438.3 \pm 12.7 \mathrm{~g}$, respectively, at the end of the study (60 days; $p<0.05$ ).

OGTT Peak values of plasma glucose, insulin, GLP-1 and GIP after the OGTTs performed basally and every 10 days following the beginning of the diets are shown in Fig. 1. Circulating levels of glucose, insulin and GIP increased logarithmically with time in HFD rats, whereas GLP-1 decreased exponentially. The effects of HFD on glucose, insulin and incretins plateaued after 60 days. In contrast, the changes in control rats were not significant.

The maximum cross-correlation $(0.87 \pm 0.12)$ between the GIP and insulin incremental responses to OGTT was observed after 40 days of HFD.

The time courses of plasma glucose and insulin together with their incremental AUCs are shown in Fig. 2. The HFD rats had significantly higher glucose levels than control rats $(p<0.0001$ at all time points), with significantly larger glucose AUCs $(p<0.005)$. Insulin levels were significantly higher in HFD rats than in control rats during the whole time course $(p<0.0001)$; insulin AUC was also significantly higher $(p<0.005)$ than in control rats.

The time courses of the incretins, together with their AUCs, are reported in Fig. 3. Basal GIP levels in HFD rats were much higher than in control rats and remained higher $(p<0.0001)$ after the oral glucose load. Accordingly, GIP AUC was significantly higher in HFD rats compared with control rats $(p<0.001)$. GLP-1 circulating levels in response to the glucose load were lower in the HFD than in the control group; therefore, also the incremental GLP-1AUC was smaller in rats fed an HFD $(p<0.05)$.

KI67-positive cells Cellular aggregates positive to KI67 were observed under the villous epithelium, inside the mucosa extending into the muscularis mucosa, only in the HFD rat group $(60 \pm 10.3 \%$ vs $1 \pm 0.02 \%$ in control rats; $p<0.001$ ), as shown in Fig. 4 .

PDX-1-positive cells To identify the cellular type in the cellular aggregates, PDX-1 positivity was assessed in sections contiguous to those positive for KI67. HFD rats showed an increased number of PDX-1-positive cells into the agglomerate structures (Fig. 5; 78 $\pm 22 \%$ PDX-1positive cells in HFD rats vs $37 \pm 12 \%$ PDX-1-positive cells in control rats that, however, were present as isolated cells within the epithelium of villi; $p<0.001$ ).

Insulin-positive cell research KI67-PDX-1-positive cells were also investigated for insulin positivity. We failed to find insulin-positive cells among KI67-PDX-1-positive cells in our sample (data not shown).

GIP-positive cells The presence of GIP-positive cells was investigated in sections contiguous to the ones previously positive for KI67 and PDX-1 (Fig. 5). GIP-positive cells were found to be clustered into cellular agglomerates in HFD rats whereas they were found to be isolated and within
Fig. 1 Time course of peak plasma glucose (a), insulin (b), GLP-1 (c) and GIP (d) after OGTT performed every 10 days from the beginning of chow diet (white bars) or HFD (black bars). Values are expressed as mean $\pm \mathrm{SD}$ in 20 rats given HFD and in 20 rats given chow diet. The fitted lines refer to the HFD only. While glucose, insulin and GIP levels increased logarithmically over time, GLP-1 decreased exponentially

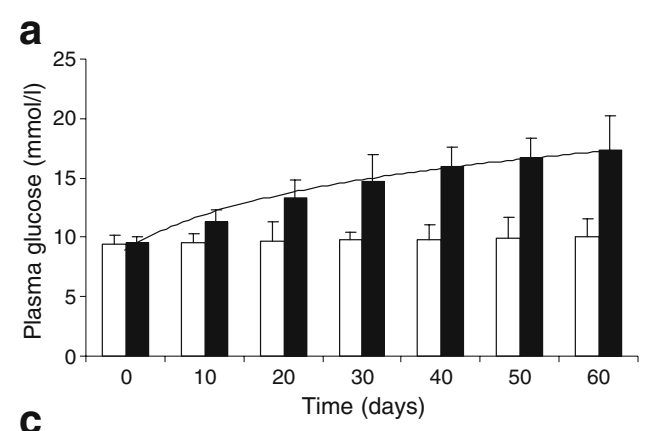

b

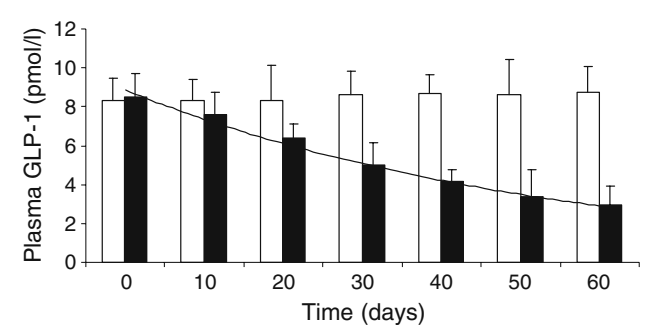

d
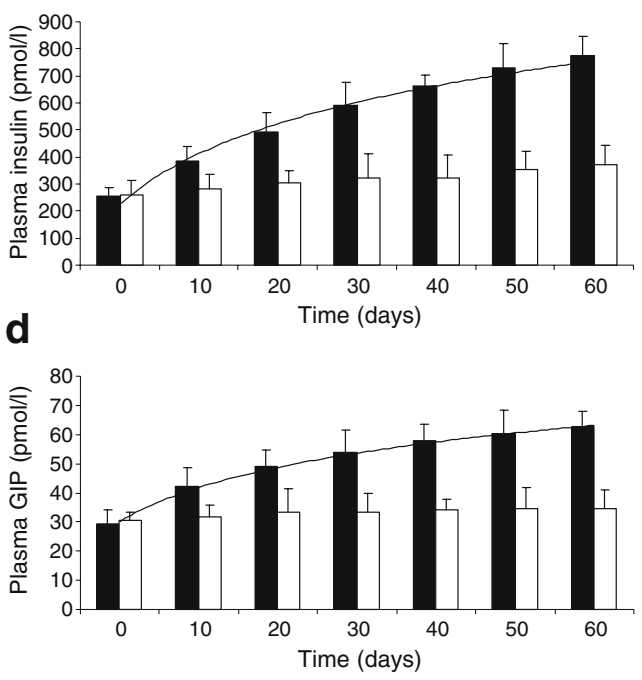
Fig. 2 Plasma glucose (a) and plasma insulin (c) concentration time course following an oral glucose gavage $(1 \mathrm{~g} / \mathrm{kg}$ body weight, $50 \%$ D-glucose solution) in 20 HFD rats (diamonds and dotted line) and in 20 control rats (squares and continuous line). In the right panel, glucose (b) and insulin (d) concentration AUCs are reported. Each sample was analysed in duplicate. In the time course figures all of the concentrations of glucose and insulin are statistically significant $(p<0.0001)$ by two-factor (time $\times$ treatment) ANOVA. In the AUC figures ${ }^{\dagger} p<0.005$ by Student's $t$ test. Values are expressed as mean $\pm \mathrm{SD}$
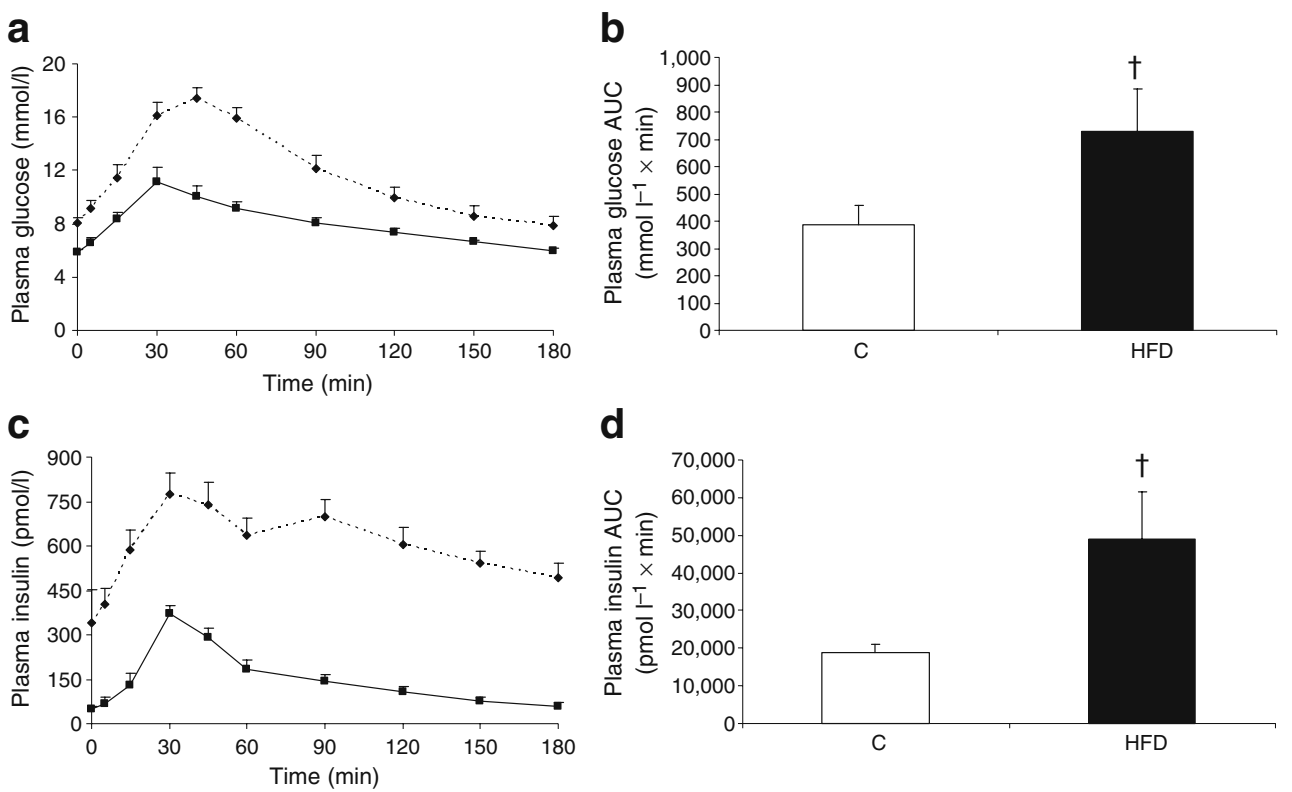

the epithelium of the villi in control rats (total number of GIP-positive cells: $500 \pm 86$ cells $/ \mathrm{mm}^{3}$ in HFD rats vs $60 \pm 13.2$ cells $/ \mathrm{mm}^{3}$ in control rats; $p<0.001$ ). In HFD rats, GIP-positive cells occupied approximately $80 \%$ of the cells positive to PDX-1.

GLP-1-positive cells The presence of GLP-1-positive cells in contiguous sections of those previously positive to KI67 and PDX-1 was also investigated. As shown in Fig. 6, GLP-1-positive cells were spread out among the submucosa, but were negative for PDX-1, even if they were significantly increased in the HFD group $\left(103 \pm 22.6\right.$ cells $/ \mathrm{mm}^{3}$ in HDF vs $63.5 \pm 33.0$ cells $/ \mathrm{mm}^{3}$ in controls; $\left.p<0.05\right)$.
Apoptosis The duodenal sections stained with haematoxylin and eosin showed inflammation with lymphomonocyte infiltration. A great increase in the number of TUNELpositive nuclei was observed after 60 days of HFD. Higher magnification of Hoechst-stained duodenal sections revealed intensely fluorescent nuclei, which is indicative of chromatin condensation, corresponding to the TUNELpositive nuclei (data not shown), as previously reported for HFD treatment for 30 and 90 days in mice [2]. Evidence for apoptosis was also confirmed by detection of fragmented DNA by ELISA; a progressive increase in optical density was found in the HFD rats compared with controls $(375 \pm 86.3 \% \mathrm{vs}$ $37 \pm 2.6 \%$ in control rats; data are optical density at $405 \mathrm{~nm}$
Fig. 3 Plasma GLP-1 ${ }_{7-36}$ (a) and plasma GIP (c) concentration time course following an oral glucose gavage $(1 \mathrm{~g} / \mathrm{kg}$ body weight, $50 \%$ D-glucose solution) in 20 HFD rats (diamonds and dotted line) and in 20 control rats (squares and continuous line). In the right panel, GLP-1 ${ }_{7-36}$ (b) and GIP (d) concentration AUCs are reported. Each sample was analysed in duplicate. In the time course figures all of the concentrations of GIP are statistically significant $(p<0.0001)$ by two-factor (time $\times$ treatment) ANOVA. In the AUC figures, ${ }^{*} p<0.05$ and $* * * p<0.001$ by Student's $t$ test. Values are expressed as mean $\pm \mathrm{SD}$ a

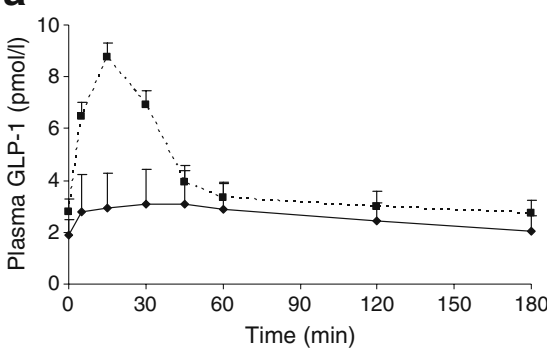

C

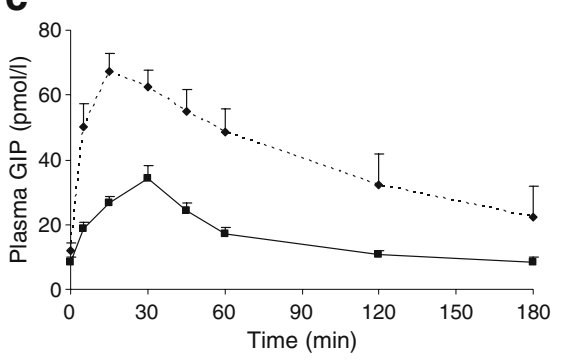

b

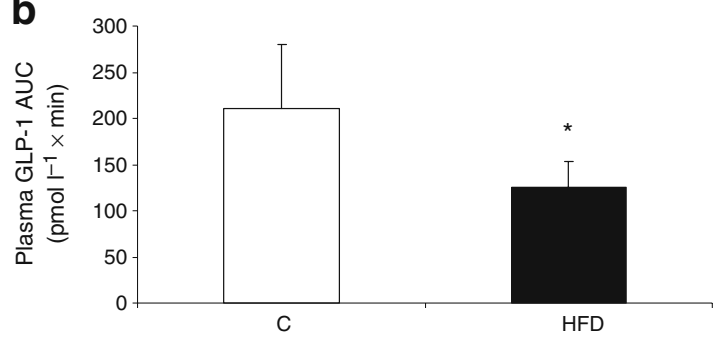

d

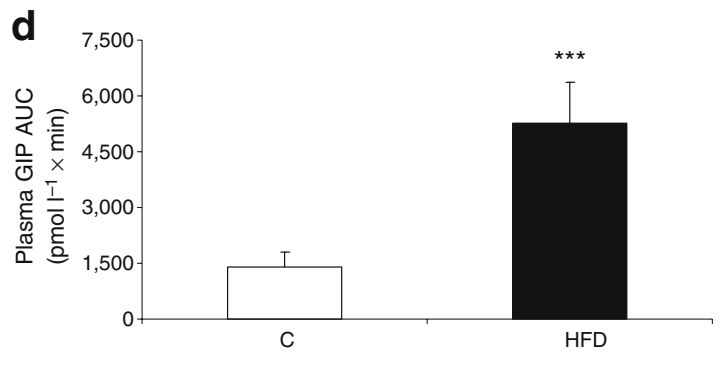


Fig. 4 Anti-KI67 immunostaining of duodenal sections from HFD rats. Ten rats given HFD and ten given the chow diet were studied; 15 sections were stained for each animal. After 60 days of HFD, KI67-positive cells, clustered into cellular agglomerates such as those found previously positive for PDX-1. a KI67-positive cells. b Nuclear reaction to Hoechst colouration in cells agglomerated. c Merged KI67 and Hoechst colouration
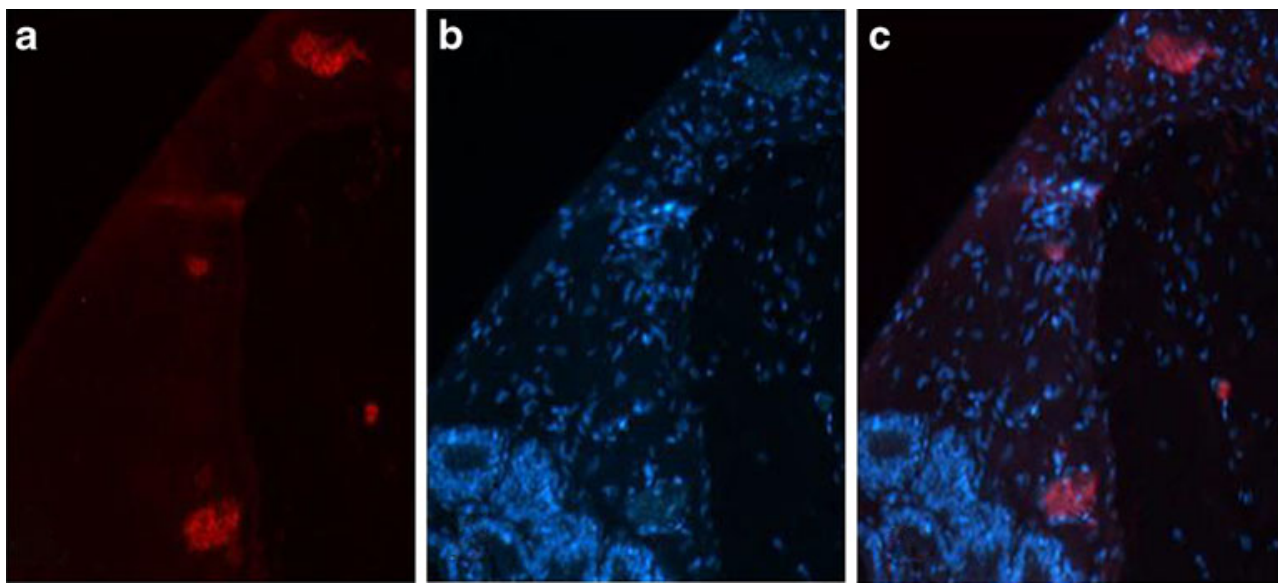

wavelength, $p<0.001)$. Apoptosis was found mainly in epithelial cells of villi.

\section{Discussion}

This is the first report showing that a saturated HFD induces proliferation of GIP-expressing cells which organise in large endocrine clusters in the duodenum of Wistar rats. The observation that these agglomerates of endocrine (PDX-1-positive), proliferating (KI67-positive) cells producing GIP are restricted to the subcrypt region suggests that they might represent undifferentiated progenitor cells. The large increase of $\mathrm{K}$ cells translates into an enhanced secretion of GIP both basally and in response to the oral glucose load compared with rats given a chow diet. The presence of a high cross-correlation between incremental response of GIP and insulin following an OGTT in the HFD group suggests that GIP drives the increase in insulin secretion. The effect of HFD in enhancing glucose circulating levels and the hormonal response to OGTT, as shown by the increase of plasma insulin and GIP peak levels, progressed with time, following a logarithmic function. The inverse of a logarithmic function is an exponential function and, in fact, GLP-1 decreased exponentially as shown in Fig. 3, suggesting that the two behaviours were opposite and possibly related to the same factor, i.e. the high-fat feeding. Furthermore, we found that the best predictors of the peak glucose levels over time were the circulating levels of insulin and GIP, almost explaining the sample variance (Table 1). In contrast, GLP-1 levels, although inversely correlated with the plasma glucose concentration, did not significantly influence the glycaemic response to the oral glucose.

In our series, GLP-1 secretion was reduced in spite of a relative increase in the number of $\mathrm{L}$ cells, suggesting that probably L cells were actively replicating at a certain time point, but highlighting the possibility that an increased rate of proliferation may paradoxically contribute to $\mathrm{L}$ cell dysfunction. There is experimental evidence demonstrating a paucity of GLP-1 release from the L cells of patients with type 2 diabetes [15-17]. It is likely that chronic hyperinsulinaemia determined by HFD, and which is also a characteristic of early diabetes and insulin resistance, may
Fig. 5 Immunofluorescence for PDX-1 (red), nuclei (blue), and GIP (green) in duodenal sections from HFD rats. Ten rats under HFD and ten under chow diet were selected randomly in the two groups; 15 sections were stained for each animal. In the upper panel the co-staining for nuclei and PDX-1 is depicted. In a,d of the lower panel it is evident the green GIP co-staining of PDX-1 positive cells, indicating that endocrine cells produce GIP. In $\mathbf{d}$ of the lower panel the immunostaining for GIP is depicted while in b,d merged Hoechst and GIP is represented
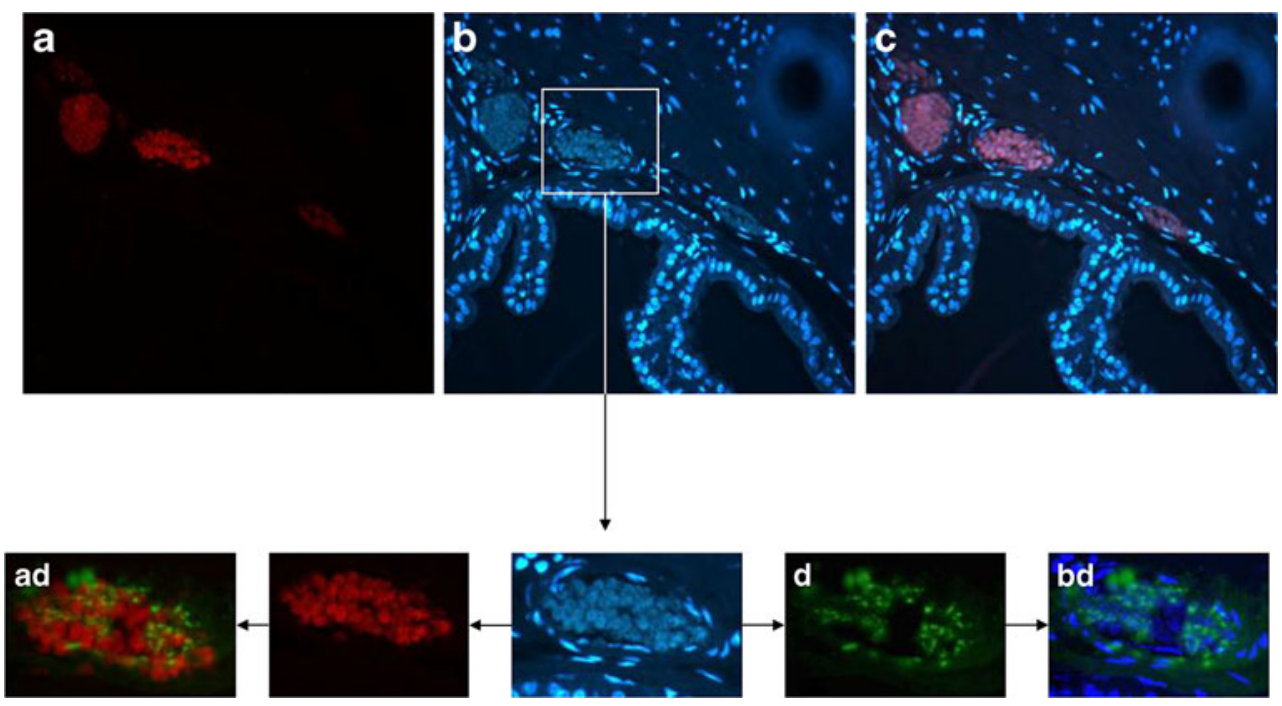
Fig. 6 GLP-1-positive cells spread out among the submucosa after the 60 days of HFD. a GLP-1-positive cells. b Nuclear reaction to Hoechst coloration. c Merged GLP-1 and Hoechst colouration. Ten rats given HFD and ten given chow diet were studied; 15 sections were stained for each animal

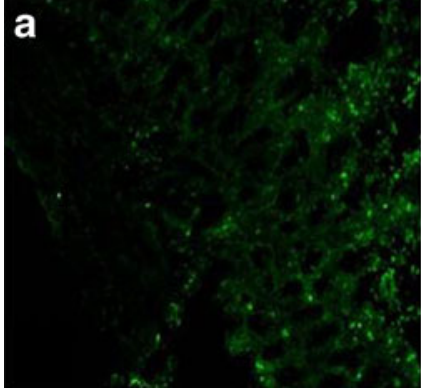

b
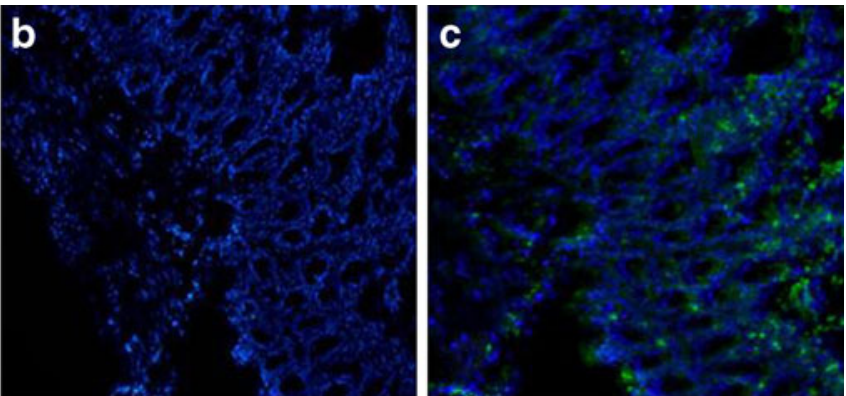

exert damaging effects upon $\mathrm{L}$ cell function via a feedforward cycle. Furthermore, in other organs, like the endocrine pancreas, of animal models of type 2 diabetes, such as MKR mice and Zucker diabetic fatty rats, beta cell dysfunction occurs despite compensatory increases in insulin content and beta cell mass mediated by hyperplasia and hypertrophy $[18,19]$.

It is well ascertained that increased consumption of energydense foods high in saturated fats is a key factor in obesity development and subsequent complications, including insulin resistance and type 2 diabetes [20]. However, the bridge between high-fat obesitogenic diet and obesity comorbidities is not fully understood. High-fat feeding, such as that used in the present study, allows intestinal K-cell hyperplasia, enhancing GIP gene expression together with intestinal GIP content, ultimately resulting in high basal and feedinginduced circulating levels of GIP [6-9]. The role played by GIP in mediating the effects of HFD, i.e. the appearance of insulin resistance and diabetes, has been elucidated by the studies of Flatt and colleagues $[8,9,11,13]$. In fact, the blockade of GIP action using a GIP antagonist [11] in mice with obesity and diabetes secondary to HFD results in a large weight loss, and in a net improvement of both insulin resistance and diabetes. Furthermore, the exclusion of the duodenum from nutrient transit after gastric bypass results in a net improvement of glucose metabolism in patients with type 2 diabetes, probably as a consequence of the lowering of GIP circulating levels [21].

However, the mechanism inducing $\mathrm{K}$ cell hyperplasia is still unknown. The gene, pancreatic duodenal homeobox-1, belongs to the ParaHox gene family of transcription factors. In mouse embryos, $P d x I$ expression marks the dorsal and ventral pancreatic buds and the duodenal endoderm between them [21] and it is maintained in the adult duodenal epithelium [22, 23]. Evidence of the involvement of $P d x 1$ in the regulation of GIP production within the enteroendocrine $\mathrm{K}$ cells of the proximal duodenum, and of the co-production of GIP and PDX1 in K cells is reported in literature [24]. It is well known that homozygosity for mutations in the $P d x l$ gene in mice affects the enteroendocrine cells in the duodenum [25-27] up to a $72 \%$ reduction in $\mathrm{K}$ cells [26]. However, GIP is generally produced by dispersed enteroendocrine $\mathrm{K}$ cells whereas in the present study we observed the formation of large endocrine clusters in the duodenal mucosa, probably meaning that under HFD the progenitor cells proliferate (as shown by the presence of KI67 marker) and differentiate towards endocrine cells (as indicated by PDX-1 positivity) which produce GIP (as suggested by GIP positivity) and aggregate in agglomerates.

The HFD promotes tissue inflammation [28] and chronic low-grade inflammation is now also recognised as a key step in the pathogenesis of obesity-induced insulin resistance and type 2 diabetes [29]. Indeed, in the present study we have observed duodenal inflammation with abundant accumulation of inflammatory cells throughout the mucosa and extending into the submucosa. Human peripheral blood monocytes, cells that are present in tissue inflammation, show the capacity to differentiate into endocrine cells [30]. In particular, neoislets can be generated from peripheral blood monocytes that show glucose-regulated insulin secretion [30].

However, the cellular agglomerates that produce GIP found inside the duodenal mucosa in these series might also derive from progenitor cells of endodermic origin which differentiate into endocrine cells under HFD stimulation. Kojima et al. [31] showed that rat intestinal stem cells, transfected with genes encoding PDX-1 and Isl-1 and successively exposed to beta cellulin, were able to produce
Table 1 Multiple regression analysis

Dependent variable: peak plasma glucose concentration after OGTT 60 days after starting HFD (GLUC60). Independent variables: peak GLP-1, GIP and insulin after OGTT at the same time period. Adjusted $R^{2}=0.90, p<0.05$

\begin{tabular}{|c|c|c|c|c|c|c|}
\hline \multirow[t]{2}{*}{ Variable } & \multirow{2}{*}{$\begin{array}{l}\text { Unstandardised coefficient } \\
\qquad \beta \pm \mathrm{SE}\end{array}$} & \multicolumn{3}{|c|}{ Standardised coefficient } & \multicolumn{2}{|c|}{ Correlation } \\
\hline & & $\beta$ & $t$ & $p$ value & Partial & Part \\
\hline (Constant) & $194.52 \pm 28.63$ & & 6.794 & 0.021 & & \\
\hline GLP-1 & $-2.56 \pm 1.75$ & -0.259 & -1.463 & 0.281 & -0.719 & -0.203 \\
\hline GIP & $2.11 \pm 0.44$ & 0.839 & 4.797 & 0.041 & 0.959 & 0.667 \\
\hline Insulin & $0.95 \pm 0.17$ & 0.845 & 5.665 & 0.030 & 0.970 & 0.788 \\
\hline
\end{tabular}


insulin and therefore to reduce plasma glucose levels after transplantation in diabetic rats.

In conclusion, with the limitation that we could not really establish a causal relationship between events, our study shows that high-fat feeding stimulates the duodenal proliferation of endocrine cells that differentiate towards $\mathrm{K}$ cell oversecretion of GIP. This progressive increment of circulating levels of GIP might represent the stimulus for insulin hypersecretion and insulin resistance, ultimately resulting in a derangement of glucose metabolism and diabetes development.

\section{References}

1. Ohtsubo K, Takamatsu S, Minowa MT, Yoshida A, Takeuchi M, Marth JD (2005) Dietary and genetic control of glucose transporter 2 glycosylation promotes insulin secretion in suppressing diabetes. Cell 123:1307-1321

2. Gniuli D, Calcagno A, Caristo ME et al (2008) Effects of a high fat diet exposure during fetal life on type 2 diabetes development in the progeny. J Lipid Res 49:1936-1945

3. Kahn SE, Hull RL, Utzschneider KM (2006) Mechanisms linking obesity to insulin resistance and type 2 diabetes. Nature 444:840-846

4. Riccardi G, Giacco R, Rivellese AA (2004) Dietary fat, insulin sensitivity and the metabolic syndrome. Clin Nutr 23:447-456

5. Bonnard C, Durand A, Peyrol S et al (2008) Mitochondrial dysfunction results from oxidative stress in the skeletal muscle of diet-induced insulin-resistant mice. J Clin Invest 118:789-800

6. Flatt PR, Bailey CJ, Kwasowski P, Swanston-Flatt SK, Marks V (1983) Abnormalities of GIP in spontaneous syndromes of obesity and diabetes in mice. Diabetes 32:433-435

7. Bailey CJ, Flatt PR, Kwasowski P, Powell CJ, Marks V (1986) Immunoreactive gastric inhibitory polypeptide and $\mathrm{K}$ cell hyperplasia in obese hyperglycaemic (ob/ob) mice fed high fat and high carbohydrate cafeteria diets. Acta Endocrinol (Copenh) 112:224-229

8. Flatt PR, Bailey CJ, Kwasowski P, Swanston-Flatt SK (1990) Effects of diets rich in sucrose, coconut fat and safflowerseed oil on the development of the obese hyperglycaemic (ob/ob) syndrome in mice. Diab Res 13:23-28

9. Ponter AA, Salter DN, Morgan LM, Flatt PR (1991) The effect of energy source and feeding level on the hormones of the enteroinsular axis and plasma glucose in the growing pig. Br $\mathrm{J}$ Nutr 66:187-197

10. Pederson RA, Brown JC (1976) The insulinotropic action of gastric inhibitory polypeptide in the perfused isolated rat pancreas. Endocrinology 99:780-785

11. McClean PL, Irwin N, Cassidy RS, Holst JJ, Gault VA, Flatt PR (2007) GIP receptor antagonism reverses obesity, insulin resistance, and associated metabolic disturbances induced in mice by prolonged consumption of high-fat diet. Am J Physiol Endocrinol Metab 293:E1746-E1755

12. Mortensen K, Petersen LL, Orskov C (2000) Colocalization of GLP-1 and GIP in human and porcine intestine. Ann NY Acad Sci 921:469-472

13. Yasuhara S, Perez ME, Kanakubo E, Yasuhara Y, Shin YS, Kaneki M et al (2000) A skeletal muscle apoptosis after burns is associated with activation of proapoptotic signals. Am J Physiol Endocrinol Metab 279:E1114-E1121

14. Gniuli D, Dalla Libera L, Caristo ME, Calvani R, Castagneto M, Mingrone G (2008) High saturated-fat diet induces apoptosis in rat enterocytes and blunts GIP and insulin-secretive response to oral glucose load. Int J Obes (Lond) 32:871-874

15. Jones IR, Owens DR, Moody AJ, Luzio SD, Morris T, Hayes TM (1987) The effects of glucose-dependent insulinotropic polypeptide infused at physiological concentrations in normal subjects and type 2 (non-insulin-dependent) diabetic patients on glucose tolerance and B cell secretion. Diabetologia 30:707-712

16. Toft-Nielsen MB, Damholt MB, Madsbad S et al (2001) Determinants of the impaired secretion of glucagon-like peptide- 1 in type 2 diabetic patients. J Clin Endocrinol Metab 86:3717-3723

17. Vilsbøll T, Knop FK, Krarup T et al (2003) The pathophysiology of diabetes involves a defective amplification of the late-phase insulin response to glucose by glucose-dependent insulinotropic polypeptide-regardless of etiology and phenotype. J Clin Endocrinol Metab 88:4897-4903

18. World Health Organization (2007) Obesity and overweight. Available from www.who.int/mediacentre/factsheets/fs311/en/index. html, accessed 1 June 2010

19. Pick A, Clark J, Kubstrup C et al (1998) Role of apoptosis in failure of beta-cell mass compensation for insulin resistance and beta-cell defects in the male Zucker diabetic fatty rat. Diabetes 47:358-364

20. Asghar Z, Yau D, Chan F, LeRoith D, Chan CB, Wheeler MB (2006) Insulin resistance causes increased beta-cell mass but defective glucose-stimulated insulin secretion in a murine model of type 2 diabetes. Diabetologia 49:90-99

21. Rubino F, Gagner M, Gentileschi P et al (2004) The early effect of the Roux-en-Y gastric bypass on hormones involved in body weight regulation and glucose metabolism. Ann Surg 240:236-242

22. Guz Y, Montminy MR, Stein R et al (1995) Expression of murine $S T F-1$, a putative insulin gene transcription factor, in beta cells of pancreas, duodenal epithelium, and pancreatic exocrine and endocrine progenitors during ontogeny. Development 121:11-18

23. Miller CP, McGehee RE Jr, Habener JF (1994) IDX-1: a new homeodomain transcription factor expressed in rat pancreatic islets and duodenum that transactivates the somatostatin gene. EMBO J 13:1145-1156

24. Jepeal LI, Fujitani Y, Boylan MO, Wilson CN, Wright CV, Wolfe MM (2005) Cell-specific expression of glucose-dependentinsulinotropic polypeptide is regulated by the transcription factor PDX-1. Endocrinology 146:383-391

25. Offield MF, Jetton TL, Labosky PA et al (1996) PDX-1 is required for pancreatic outgrowth and differentiation of the rostral duodenum. Development 122:983-995

26. Larsson LI, Madsen OD, Serup P, Jonsson J, Edlund H (1996) Pancreatic-duodenal homeobox 1: role in gastric endocrine patterning. Mech Dev 60:175-184

27. Jonsson J, Carlsson L, Edlund T, Edlund H (1994) Insulinpromoter-factor 1 is required for pancreas development in mice. Nature 371:606-609

28. Todoric J, Löffler M, Huber J et al (2006) Adipose tissue inflammation induced by high-fat diet in obese diabetic mice is prevented by $n-3$ polyunsaturated fatty acids. Diabetologia 49:2109-2119

29. Fernández-Real JM, Ricart W (1999) Insulin resistance and inflammation in an evolutionary perspective: the contribution of cytokine genotype/phenotype to thriftiness. Diabetologia 42:1367-1374

30. Ruhnke M, Ungefroren H, Nussler A et al (2005) Differentiation of in vitro modified human peripheral blood monocytes into hepatocytelike and pancreatic islet-like cells. Gastroenterology 128:1774 1786

31. Kojima H, Nakamura T, Fujita Y et al (2002) Combined expression of pancreatic duodenal homeobox 1 and islet factor 1 induces immature enterocytes to produce insulin. Diabetes 51:1398-1408 